\title{
Morphometric Study of the Lumbar Spray in the African Black West Subject: Interest in Surgery. About a CT Scans of 170 Cases in Ouagadougou (Burkina Faso)
}

\author{
Mohamed Tall ${ }^{1}$, Mamoudou Sawadogo ${ }^{*}$, Amadou Ndiasse Kassé2, Adama Ouédraogo1, \\ Hervé Pilabré ${ }^{1}$, Issouf Savadogo ${ }^{1}$, Ousséni Diallo ${ }^{3}$, Rabiou Cissé ${ }^{3}$ \\ ${ }^{1}$ Department of Orthopedics-Traumatology, Yalgado Ouedraogo University Hospital, Ouagadougou, Burkina Faso \\ ${ }^{2}$ Grand Yoff General Hospital, Dakar, Senegal \\ ${ }^{3}$ Department of Radiology, Yalgado Ouedraogo University Hospital, Ouagadougou, Burkina Faso \\ Email: *smamoudou@hotmail.com
}

How to cite this paper: Tall, M., Sawadogo, M., Kassé, A.N., Ouédraogo, A., Pilabré, H., Savadogo, I., Diallo, O. and Cissé, R. (2018) Morphometric Study of the Lumbar Spray in the African Black West Subject: Interest in Surgery. About a CT Scans of 170 Cases in Ouagadougou (Burkina Faso). Open Journal of Orthopedics, 8, 190-199. https://doi.org/10.4236/ojo.2018.85022

Received: March 14, 2018

Accepted: May 21, 2018

Published: May 24, 2018

Copyright () 2018 by authors and Scientific Research Publishing Inc. This work is licensed under the Creative Commons Attribution International License (CC BY 4.0).

http://creativecommons.org/licenses/by/4.0/

\begin{abstract}
Objective: Our study aimed at analyzing the morphometry of the lumbar spine at the CT scan and to specify its interest in spinal surgery. Methodology: This was a prospective study conducted from May 1, 2011 to July 31, 2011 in Ouagadougou. During this study, 170 patients aged $45.3 \pm 12.5$ years old with a sex ratio of 1.15 received a CT scan. The symptomatology was dominated by low back pain (60.6\%) and lumbar osteoarthritis was the most common etiology (51.8\%). The measurements were performed directly on axial and sagittal sections of the lumbar spine with measurement of the vertebral body, and pedicles. Results: The vertebral body increased forward from 24.9 $\mathrm{mm}$ in $\mathrm{L} 1$ to $26.7 \mathrm{~mm}$ in L5 and decreasing backwards from $26.6 \mathrm{~mm}$ in $\mathrm{L} 1$ to $23.4 \mathrm{~mm}$ in L5. The transverse diameter of the vertebral body ranged from 37 $\mathrm{mm}$ in $\mathrm{L} 1$ to $47.4 \mathrm{~mm}$ in L5 and the anteroposterior diameter of $27.6 \mathrm{~mm}$ in L1 to $33.5 \mathrm{~mm}$ in L5. The lumbar canal had an anteroposterior diameter that ranged from $15.9 \mathrm{~mm}$ in $\mathrm{L} 1$ to $15.5 \mathrm{~mm}$ in $\mathrm{L} 5$ and an interpedicular distance increasing from $21.54 \mathrm{~mm}$ in $\mathrm{L} 1$ to $28.42 \mathrm{~mm}$ in L5. The pedicle decreased in length from L1 $(9.5 \mathrm{~mm})$ to $\mathrm{L} 5(6.5 \mathrm{~mm})$ while its width increased from 6.9 $\mathrm{mm}$ in $\mathrm{L} 1$ to $15.1 \mathrm{~mm}$ in L5. Its axis formed, with respect to the sagittal plane, an increasingly obtuse angle varying from 16.3 degrees in L1 to 29.1 degrees in L5 and with respect to the upper plate of the vertebral body, an increasingly acute angle (14, 2 degrees in L1 at 8.8 degrees in L5). Conclusion: The dimensions of the lumbar vertebrae in our series differ from those noted in Western populations in that they have a smaller vertebral body, a shorter, wider pedicle with an angle of inclination of the pedicle compared to the sa-
\end{abstract}


gittal and horizontal planes more obtuse.

\section{Keywords}

Morphometry, Lumbar Spine, Surgery

\section{Introduction}

Considered by many authors as a public health problem [1] [2] [3] [4], low back pain is a common reason for consultation and disability. It is the leading cause of disability and incapacity to work before age 45 in France [2]. The management of spinal pathology, although dominated by medical treatment, sometimes gives rise to surgical indications. The pedicle, which is the junction between the posterior and anterior parts of the spine, is a key element for the surgical management of vertebral affections. Indeed, the use of pedicle screws has become the mainstream of lumbar fusion surgery. A precise knowledge of the anatomy of the vertebrae that make up the lumbar spine is therefore necessary for the surgeon for correct positioning of the pedicle screws. To date, many studies on lumbar spine morphometry have been conducted in various populations [5]-[10], but none in West Africa. These studies have shown that there are sometimes variations in the anatomy of the lumbar vertebra depending on the type of population. The purpose of this work is to study the morphometry of the lumbar spine in the West African subject and to draw the interest in surgery.

\section{Materials and Method}

This was a prospective, descriptive and analytical study that included $170 \mathrm{CT}$ scans of the lumbar spine performed between May 1, 2011 and July 31, 2011. Included were all patients who performed a CT scan during the study period for back pain. CT performed for another reason were not taken into account. The average age of the patients was $45.3 \pm 12.5$ years with a sex ratio of 1.15 . The symptomatology was dominated by low back pain, sciatica or lumbosciatica.

The measurements were performed directly on a scanner of 8 bars on axial and sagittal sections.

Measuring technique

The acquisition of images was done by two (02) senior technicians in medical imaging and measurements by the same radiologist physician.

The height of the vertebral bodies was measured forwards and backwards on a mid-sagittal section as well as the intersomatic space.

On an axial section passing through the pedicles at half height, the following parameters were measured:

1) the transverse diameter (TD) and the anteroposterior diameter (APD) of the vertebral body at the median level.

2) the anteroposterior diameter of the lumbar canal on the median line. 
3) the transverse diameter of the lumbar canal designated by the interpedicular distance, measured at the isthmic level.

4) the length of the pedicle measured from the posterior rim of the vertebral body to its entry into the blades and transverse processes.

5) the transverse diameter or width of the pedicle measured at its isthmus, that is to say at the narrowest part.

6) the alpha angle of inclination of the axis of the pedicle with respect to the median sagittal plane determined by a median passing through the vertebral body, the lumbar canal and the spinous process behind and a line parallel to the axis of the pedicle; it divides in its middle.

7) the beta angle of inclination of the pedicle relative to the horizontal plane measured on a sagittal section passing through the pedicle and formed by a segment parallel to the upper plate of the vertebral body and another segment passing through the middle of the height of the pedicle.

The different measurements have been shown in the figures below.

The data was analyzed with Sphinx software version 5.0.12 and Microsoft Office 2007 Excel spreadsheet. Comparisons of proportions and averages were performed using the $\chi^{2}$ and Student tests with a significance level of less than $5 \%$.

\section{Results}

1) The dimensions of the vertebral body (Figure 1 and Figure 2)

The anterior height increased from L1 to L5 and was significantly greater in men than in women $(p<0.001)$. Posterior height decreased from L1 to L5 and was significantly higher in men than in women $(p<0.001)$. The transverse and anteroposterior diameters increased significantly from L1 to L5 with mean values significantly lower in women than in men $(p<0.001)$. These different dimensions are shown in Table 1.

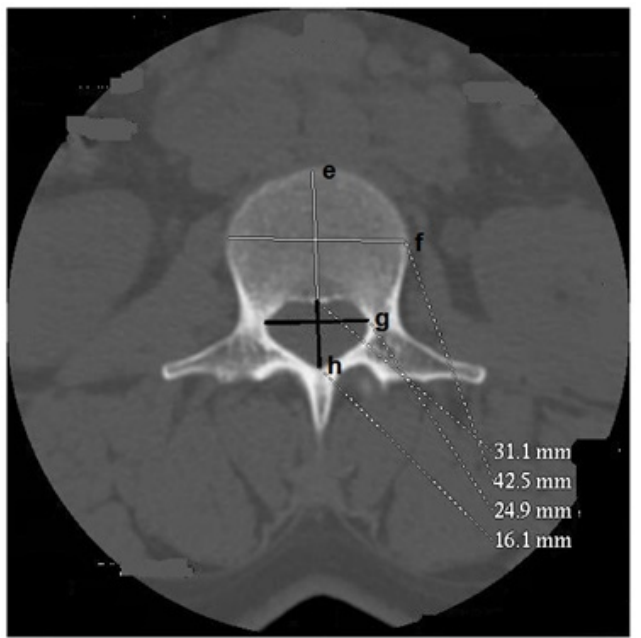

Figure 1. Axial section of the L3 lumbar vertebra passing through the pedicles (body and spinal canal dimensions). e: anteroposterior diameter of the vertebral body (DAPCV); $\mathrm{f}$ : transverse diameter of the vertebral body (DTCV); g: interpedicular distance of the spinal canal (DIP); h: anteroposterior diameter of the spinal canal (DAPCR). 

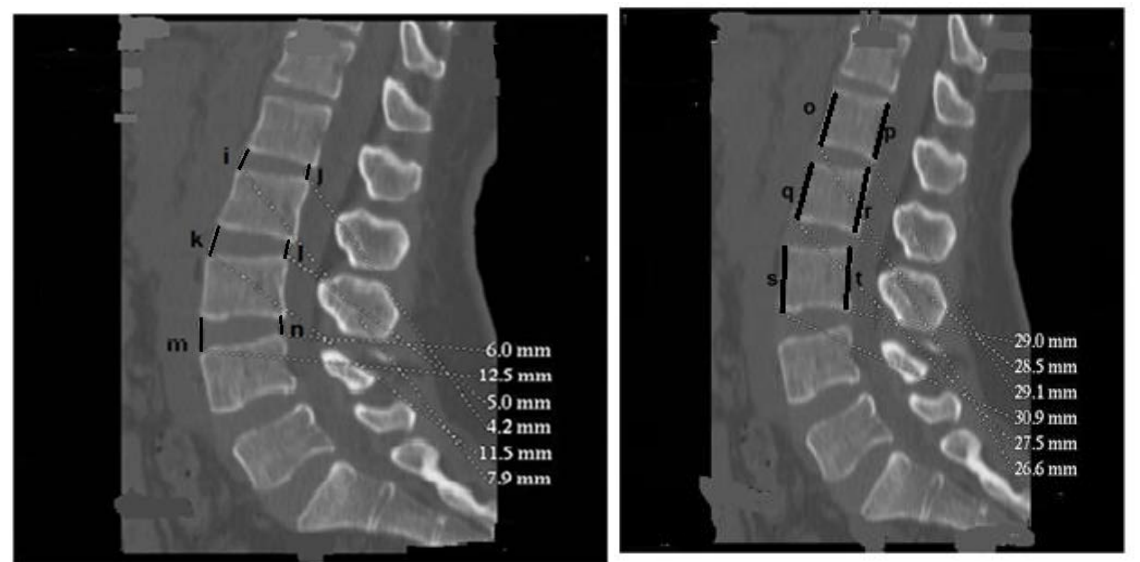

Figure 2. Mid sagittal section of the lumbar spine. i, k, m: heights of the anterior intersomatic space; $j, 1, n$ : heights of posterior intersomatic space; o, q, s: anterior heights of the vertebral bodies; $\mathrm{p}, \mathrm{r}$, t: posterior heights of the vertebral bodies.

Table 1. Vertebral body dimensions (in $\mathrm{mm}$ ).

\begin{tabular}{ccccccccccccc}
\hline & \multicolumn{4}{c}{ Ant height VB } & \multicolumn{4}{c}{ Post height VB } & \multicolumn{3}{c}{ Transverse diameter VB } & \multicolumn{3}{c}{ Ant-post diameter VB } \\
\cline { 2 - 13 } & M & W & Ave & M & W & Ave & M & W & Ave & H & W & Ave \\
\hline L1 & 25.4 & 24.3 & 24.9 & 27.5 & 25.6 & 26.6 & 38.2 & 35.7 & 37.0 & 28.7 & 26.4 & 27.6 \\
L2 & 26.4 & 25.4 & 25.9 & 27.7 & 26.7 & 27.0 & 39.1 & 36.5 & 37.9 & 30.1 & 27.8 & 29.0 \\
L3 & 26.5 & 25.6 & 26.1 & 27.1 & 25.7 & 26.5 & 40.9 & 37.8 & 39.5 & 31.5 & 29.0 & 30.3 \\
L4 & 26.5 & 25.6 & 26.1 & 25.7 & 24.4 & 25.1 & 43.7 & 40.3 & 42.1 & 32.7 & 30.1 & 31.5 \\
L5 & 27.3 & 25.9 & 26.7 & 23.9 & 22.7 & 23.4 & 49.1 & 45.5 & 47.4 & 34.6 & 32.2 & 33.5 \\
\hline
\end{tabular}

VB: vertebral body; ant: anterior; post: posterior; M: man; W: woman; Ave: average regardless of sex.

\section{2) Dimensions of the spinal canal}

The anteroposterior diameter varied very little from L1 to L5, and there was no significant difference in sex $(p=0.3)$ (Figure 1). The interpedicular distance gradually increased from L1 to L5 with significantly higher values in men than in women $(p<0.001)$. These values are shown in Table 2 below.

\section{3) Pedicle dimensions (Table 3 )}

The length of the pedicle decreased from L1 to L5 and there was no significant difference between the left and the right or between the man and the woman ( $p$ $=0.1$ ). Pedicle width increased from L1 to L5 and was the same between left and right. Values in men were significantly higher than in women $(p<0.001)$. The alpha angle increased from L1 to L5 (Figure 3 ). There was no significant difference between the left and right or between the man and the woman $(p=0.07)$. The beta angle (Figure 4) decreased from L1 to L5. The difference between the man and the woman was not statistically significant $(p=0.3)$.

\section{Comments and Discussion}

In our series, the anterior height of the vertebral body increased from L1 to L5 while the posterior height decreased from L2 to L5. In both cases, the values in 
Table 2. Dimensions of the spinal canal and horizontal angle of the pedicle.

\begin{tabular}{cccccccccc}
\hline \multicolumn{8}{c}{$\begin{array}{c}\text { Ant-post diameter of SC } \\
\text { (mm) }\end{array}$} & \multicolumn{8}{c}{$\begin{array}{c}\text { Interpedicular distance } \\
\text { SC }\end{array}$} & $\begin{array}{c}\text { Angle pedicle axis sup plateau of VB } \\
\text { (degree) }\end{array}$ \\
\hline & M & W & Ave & M & W & Ave & M & W & Ave \\
\hline L1 & 15.8 & 16.1 & 15.9 & 22.2 & 20.8 & 21.5 & 14.0 & 14.5 & 14.2 \\
L2 & 14.9 & 15.4 & 15.1 & 22.5 & 21.1 & 21.8 & 12.3 & 13.4 & 13.1 \\
L3 & 14.3 & 14.8 & 14.5 & 23.3 & 22.1 & 22.7 & 11.7 & 12.0 & 11.8 \\
L4 & 14.7 & 14.9 & 14.8 & 24.6 & 23.3 & 24.0 & 10.2 & 10.3 & 10.3 \\
L5 & 15.6 & 15.4 & 15.5 & 28.7 & 27.6 & 28.2 & 8.7 & 8.9 & 8.8 \\
\hline
\end{tabular}

SC: spinal canal.

Table 3. Dimensions of the pedicle.

\begin{tabular}{cccccccccc}
\hline & \multicolumn{3}{c}{ Pediclelength } & \multicolumn{3}{c}{ Pediclewidth } & \multicolumn{3}{c}{ Alpha angle of the pedicle } \\
\hline & M & W & Ave & M & W & Ave & M & W & Ave \\
\hline L1 R & 9.3 & 9.6 & 9.5 & 7.3 & 6.5 & 6.9 & 16.3 & 16.2 & 16.3 \\
L1 L & 9.4 & 9.6 & 9.5 & 7.3 & 6.5 & 6.9 & 16.3 & 16.2 & 16.3 \\
L2 R & 8.7 & 8.9 & 8.8 & 8.1 & 7.1 & 7.6 & 18.4 & 17.9 & 18.2 \\
L2 L & 8.7 & 8.9 & 8.8 & 8.1 & 7.0 & 7.6 & 18.4 & 17.9 & 18.2 \\
L3 R & 7.9 & 8.2 & 8.1 & 10.2 & 8.8 & 9.6 & 21.1 & 20.6 & 20.8 \\
L3 L & 8.0 & 8.3 & 8.1 & 10.2 & 8.8 & 9.6 & 21.1 & 20.6 & 20.8 \\
L4 R & 7.1 & 7.4 & 7.2 & 12.5 & 11.2 & 11.9 & 23.7 & 23.2 & 23.5 \\
L4 L & 7.2 & 7.4 & 7.3 & 12.5 & 11.2 & 11.9 & 23.7 & 23.3 & 23.5 \\
L5 R & 6.5 & 6.4 & 6.4 & 15.5 & 14.6 & 15.1 & 28.9 & 29.3 & 29.1 \\
L5 L & 6.5 & 6.4 & 6.5 & 15.5 & 14.7 & 15.1 & 28.9 & 29.3 & 29.1 \\
\hline
\end{tabular}

R: right; L: left.
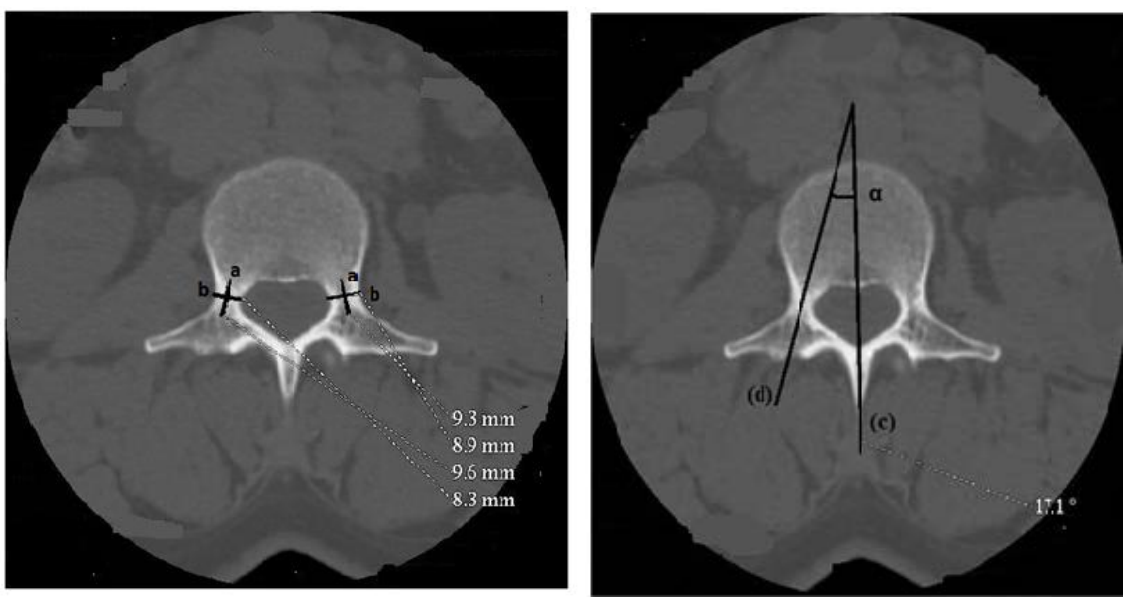

Figure3. Axial section of the lumbar vertebra L3 passing through the pedicles (dimensions of the pedicle). a: pedicle length; b: width of the pedicle; c: medio-sagittal line passing through the vertebral body, the spinal canal and the spinous process behind; d: sagittal axis of the pedicle passing through its middle; $\alpha$ : angle of inclination of the axis of the pedicle with respect to the sagittal plane.

women were lower than those found in men.

D. Fang et al. [5] in their series on the CT scan of the lumbar spine of Chinese 


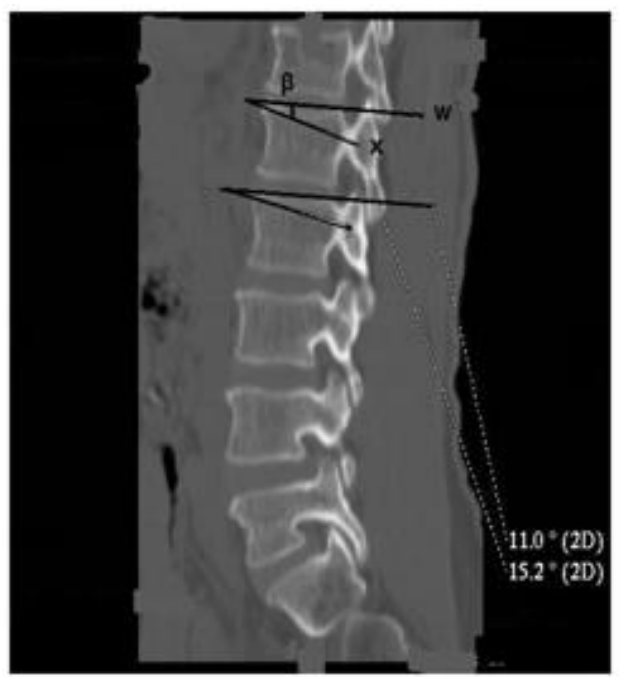

Figure 4. Sagittal section of the lumbar spine passing through the pedicles (beta angle). w: tangent to the upper plate of the vertebral body; $x$ : axis of inclination of the vertebral pedicle on the horizontal; $\beta$ : angle of inclination of the pedicle axis with respect to the horizontal plane.

populations and H. S. Karabekir et al. [6] in Turkey in their study of the morphometry of the lumbar vertebrae noted results quite close to ours. The differences in values observed are due to the inaccuracies related to the measurements and the methods used.

Unlike the literature that lumbar vertebrae are higher forwards than behind [6], our first two vertebrae are significantly higher behind than in front. It is only at the level of the last two vertebrae that the anterior height prevails over the posterior height.

In our series, the transverse and anteroposterior diameters of the vertebral body were larger in men than in women and increased significantly from L1 to L5. S. Eisentein in South Africa [10] through a retrospective study of the measurement of lumbar vertebrae of cadavers composed of blacks and Caucasians reached the same conclusions with values comparable to ours and did not note any significant difference according to race. D. Fang et al. [5] in their series noted results superimposable to ours except in L5 in men. However, for women, the transverse and anteroposterior diameters were slightly smaller than those obtained in our study.

Other authors such as Karabekir [6] in Turkey and Singh [11] in USA found in their series, similar results but that overall remained significantly higher than those noted in our series. Indeed, in the Karabekir series, TD and APD ranged from: TD: $48.9 \mathrm{~mm}$ in L1 to $57.4 \mathrm{~mm}$ in L5 in women and $48.6 \mathrm{~mm}$ in L1 to 54.5 $\mathrm{mm}$ in L5 in men. The APD: $33.4 \mathrm{~mm}$ in L1 at $39.0 \mathrm{~mm}$ in L5 in women and $35.6 \mathrm{~mm}$ in $\mathrm{L} 1$ at $39.6 \mathrm{~mm}$ in L5 in man.

In Sing's study [11]: the TD ranged from $51.3 \mathrm{~mm}$ in $\mathrm{L} 2$ to $53.9 \mathrm{~mm}$ in L5 and the APD ranged from $38.1 \mathrm{~mm}$ in $\mathrm{L} 2$ to $39 \mathrm{~mm}$ in $\mathrm{L} 5$.

These differences in results could be explained on the one hand by the fact 
that these authors used the MRI which is more precise than the CT and in addition, their results related to strictly healthy vertebrae. On the other hand one could think of racial variations. The TD and APD vertebral body would be larger in the Caucasian than in the African black in which they are identical to those noted in Asian [5].

In our series, there was a progressive increase in the interpedicular distance of the spinal canal from L1 to L5. It was greater in men than in women. The anteroposterior diameter showed no significant difference by sex and was nearly constant from L1 to L5.

Serarslan [11] in Turkey through a 2005 study of thoracolumbar spine morphometry in sickle cell subjects claimed that the interpedicular distance in their series was greater than that of Nigerians, Saudi Arabians, and Koreans, but was lower compared to that of the Spanish, Indian and Turkish non-sickle cell populations. The lumbar canal in our series was narrower than that noted in Turkish sickle cell patients, in whom it is even narrower than that of Turks without sickle cell disease. One might think that the lumbar canal would be narrower in the African black subject than in the white subject. This could partly be explained by the shorter pedicles found in our series.

Eisenstein in South Africa [10] through his study on morphometry of the lumbar spine comparing black and white subjects noted results superimposable ours.

Fang [5] for his part concluded that compared to whites, Asians had a smaller vertebral body and there was a difference in the shape of the lumbar canal. These results are quite close to ours except in L5 where their value was much smaller. The differences in values observed at the anteroposterior diameter could be explained by the high number of cases of lumbar stenosis in our series, especially since it is this diameter that is most affected in case of stenosis of the canal.

The pedicle has been the subject of numerous studies in various human populations in order to provide surgeons with a precise knowledge of its anatomy and from there, to minimize the risks inherent in the technique of transpedicular fixation fusion.

Thus in our series, the length of the pedicle decreased from L1 to L5 and there was no significant difference by sex or vertebral side. The width, unlike the length, increased significantly from L1 to L5. There was no significant difference between left and right. However, it was greater in men than in women. The alpha angle of inclination of the pedicle axis with respect to the sagittal plane became more and more obtuse from L1 to L5. There was no significant difference between left and right or between the man and the woman. As for the angle beta of the axis of the pedicle with respect to the upper plate of the vertebral body, it became more and more acute from L1 to L5 and varied on average from 14.2 degree in L1 to 8.8 degree in L5. The difference between the man and the woman was not statistically significant although the values in humans seem smaller.

Maaly [9] in Egypt performed a morphometric study of the lumbar pedicle on 
75 patients. He noted that the pedicle was $5 \mathrm{~cm}$ long on average and almost constant from L1 to L5. These values are significantly larger than those obtained in our series. However, for the width and alpha angle of the pedicle their results were similar to ours except in L5 where their pedicle was significantly wider. The difference from our results in the length of the pedicle is related to the fact that Maaly [9] in his study considered this length from the point of entry of the pedicle screw to the anterior edge of vertebral body (pedicle length plus anteroposterior diameter of the vertebral body). As the pedicle becomes shorter and the vertebral body becomes larger and larger from front to back from L1 to L5, we can then understand the almost constant length of their pedicle. This leads us to conclude that the pedicle in our series has the same characteristics as that of the Egyptians, especially since we used lumbar pain, a comparable pedicle and the same methodology as Maaly [9] et al. inspired by that used by Zindrick [12].

Olsewski [8] in the US reported in his series on lumbar spine morphometry in 51 patients, similar results to ours in terms of vertebral width, but with a higher alpha angle. The variations observed with our results, especially at the level of the first two vertebrae in humans, could be explained by the small size of their sample, especially since some values in our sample are beyond their average. The beta angle in their study was also more acute and almost constant from L1 to L5 with mean values of 7 degrees in men and 6 degrees in women.

Other authors in their series have found a pedicle that has characteristics totally different from ours. Thus Karabekir [6] in Turkey found a narrower and longer pedicle, a more acute alpha angle and varying very little from L1 to L5 compared to our results. There was no difference by sex or vertebral side. The difference with our results could be explained by racial variations in the anatomy of the pedicle. One could then think that the pedicle of African black has an angle more obtuse with respect to the sagittal plane than that of Westerners. This is a particularity to take into account when placing the pedicle screws. These screws should be inserted obliquely backwards and forwards, from outwards inwards and at an angle ranging from 14 degrees in L1 to 8 degrees in L5 in the horizontal plane and 16 degrees in L1 at 29 degrees in L5 in the sagittal plane contrary to Roy Camille's technique of aiming straight ahead. This diversity in the anatomy of the pedicle reflects the need to adapt the pedicle screws not only according to the type of population but also according to the vertebral level concerned. In our series the screws should be of increasing diameter from L1 to L5. Their dimensions could be chosen according to those proposed by Maaly [9] who in their study also evaluated the endo-osseous thickness of the pedicle which, moreover, was lower than that of the Chinese except in L5. They estimated that the diameter of the pedicle screws should be $1 \mathrm{~mm}$ more than the endo-osseous thickness of the corresponding pedicle. They then noted that the screw diameter should be respectively from L1 to L5 of 6.0; 7.0; 8.5; 8.5 and 9.5 $\mathrm{mm}$ in humans and 5.0; 5.0; 6.5; 6.5 and $8 \mathrm{~mm}$ in women and that the length should be less than $45 \mathrm{~mm}$ in women and less than $50 \mathrm{~mm}$ in men without tak- 
ing into account the vertebral level concerned.

MitraSajal [13] in 2002 pointed out in a study of lumbar pedicle morphometry in Indians that a $5 \mathrm{~mm}$ screw should be used in the upper lumbar spine (L1-L2) while in the low (L3-L4) a $6 \mathrm{~mm}$ screw is required.

Karabekir in Turkey [6] in his study estimated the diameter of the pedicle screw at least $5 \mathrm{~mm}$.

\section{Conclusion}

The morphometric study of the lumbar vertebrae allowed us to notice a vertebral body and a lumbar canal identical to those of the Asian and which seemed to be smaller compared to those found in the Westerners and the Americans. The pedicle in our series has the same characteristics as that of the Egyptians and Asians; however, it is shorter, wider, with an angle of insertion with respect to the sagittal plane more obtuse than that of the Westerners. The direction of the screws should be oblique from back to front, from outside to inside, at an angle ranging from 16 degrees in L1 to 29 degrees in L5 in the sagittal plane and from 14 degrees to 8 degrees in the horizontal plane contrary to the Roy Camille's technique of aiming straight ahead. Measurements made on strictly healthy vertebrae would have allowed us to obtain more reliable results. Also an additional measurement of the endosseous thickness of the pedicle and the path of the screw from its point of entry to the anterior rim of the vertebral body would allow choosing appropriately the size of the pedicle screws.

\section{References}

[1] Cheour, E., Hamdi, W., Tekaya, R., Hamza, S., Kerkeni, S., Elleuch, M., Sahli, H., Khosrof, M. and Sellami, S. (2007) Common Lumbosciatic Syndrome of the Elderly. Report of 67 Cases. La Tunisie Medicale, 85, 549-552.

[2] Haumesser, D., Becker, P., Grosso-lebon, B. and Weill, G. (2004) Medical, Social and Economic Aspects of the Management of Chronic Low Back Pain. Pratiques et organisation des soins, 35, 27-36.

[3] Ntsiba, H., Bileckot, R., Kala, J.C. and Bregeon, C. (1993) 200 Cases of Sciatica in the Congolese Subject. Revue du rhumatisme et des maladies ostéo-articulaires, 60 , 737.

[4] Younes, M., Bejia, I., Aguir, Z., Laetaief, M., Hassen-Zrour, S. and Touzi, M. (2006) Prevalence and Factors Associated with Common Sciatica in a Tunisian Urban Population. Revue Du Rhumatisme, 73, 927-931. https://doi.org/10.1016/j.rhum.2005.10.024

[5] Fang, D., Cheung, K.M., Ruan, D. and Chan, F.L. (1994) Computed Tomographic Osteometry of the Asian Lumbar Spine. Journal of Spinal Disorders, 7, 307-316. https://doi.org/10.1097/00002517-199408000-00004

[6] Karabekir, H.S., Gocmen-Mas, N., Edizer, M., Ertekin, T., Yazici, C. and Atamturk, D. (2011) Lumbar Vertebra Morphometry and Stereological Assesment of Intervertebral Space Volumetry: A Methodological Study. Annals of Anatomy, 193, 231-236. https://doi.org/10.1016/j.aanat.2011.01.011

[7] Hasegawa, T., An, H.S., Haughton, V.M. and Nowicki, B.H. (1995) Lumbarforami- 
nalstenosis: Criticalheights of the Intervertebraldiscs and Foramina. A Cryomicrotome Study in Cadavera. The Journal of Bone and Joint Surgery. American Volume, 77, 32-38. https://doi.org/10.2106/00004623-199501000-00005

[8] Olsewski, J.M., Simmons, E.H., Kallen, F.C., Mendel, F.C., Severin, C.M. and Berens, D.L. (1990) Morphometry of the Lumbar Spine: Anatomical Perpectives Related to Transpedicular Fixation. The Journal of Bone and Joint Surgery. American Volume, 72, 541-549. https://doi.org/10.2106/00004623-199072040-00011

[9] Maaly, M.A., Saad, A. and Houlel, M.E.E. (2010) Morphological Measurements of Lumbar Pedicles in Egyptian Population Using Computerized Tomography and Cadaver Direct Caliber Measurements. The Egyptian Journal of Radiology and Nuclear Medicine, 41, 475-481. https://doi.org/10.1016/j.ejrnm.2010.10.002

[10] Eisenstein, S. (1977) The Morphometry and Pathological Anatomy of the Lumbar Spine in South African Negroes and Caucasoids with Specific Reference to Spinal Stenosis. The Journal of Bone and Joint Surgery. British Volume, 59, 173-180. https://doi.org/10.1302/0301-620X.59B2.873978

[11] Serarslan, Y., Kalaci, A., Ozkan, C., Doğramaci, Y., Cokluk, C. and Yanat, A.N. (2010) Morphometry of the Thoraco Lumbar Vertebrae in Sickle Cell Disease. Journal of Clinical Neuroscience, 17, 182-186. https://doi.org/10.1016/j.jocn.2009.05.010

[12] Zindrick, M.R., Wiltse, L.L., Doornik, A., Widell, E.H., Knight, G.W., Patwardhan, A.G., Thomas, J.C., Rothman, S.L. and Fields, B.T. (1987) Analysis of the Morphometric Characteristics of the Thoracic and Lumbar Pedicles. Spine (Phila Pa 1976), 12, 160-166. https://doi.org/10.1097/00007632-198703000-00012

[13] Mitra, S.R., Datir, S.P. and Jadhav, S.O. (2002) Morphometric Study of the Lumbar Pedicle in the Indian Population as Related to Pedicular Screw Fixation. Spine (Phila Pa 1976), 27, 453-459. https://doi.org/10.1097/00007632-200203010-00004 